\title{
Efectos de un programa de ejercicios sobre el dolor lumbar en trabajadores de oficina
}

\author{
Margareth Lorena Alfonso-Mora* \\ Yeni Paola Romero-Ardila** \\ Eliana Marcela Montaño-Gil**
}

\begin{abstract}
* Fisioterapeuta. Docente Facultad de Enfermería y Rehabilitación. Departamento Movimiento Corporal Humano. Universidad de La Sabana. Bogotá. Colombia

** Fisioterapeuta. Universidad de La Sabana. Bogotá. Colombia.

Correspondencia: Srta. Margareth Lorena Alfonso Mora. Dirreción: campus del puente del común, Km 7, autopista Norte de Bogotá. Chía. Cundinamarca. Colombia. Teléfono: +578615555. Correo electronico: margarethalmo@unisabana.edu.co
\end{abstract}

\section{RESUMEN}

Introducción: el dolor lumbar es una condición con mayor índice de consulta en los servicios de salud. En trabajadores de oficina también existe una alta prevalencia, por lo tanto es necesario generar planes de intervención desde la prevención primaria para mejorar la condición de salud de esta población. Objetivo: determinar los efectos de un programa de ejercicio sobre el dolor lumbar en trabajadores de oficina. Materiales y métodos: se realizó un programa de ejercicio en 17 trabajadores de oficina en una empresa de Bogotá durante cuatro semanas basado en el fortalecimiento de los músculos abdominales, profundos del cuello y el estiramiento de la cadena recta posterior. Se evaluó la evolución del dolor lumbar y la incapacidad laboral por medio del cuestionario de Roland Morris, los instrumentos se aplicaron al finalizar las cuatro semanas de intervención con seguimiento a los seis meses. Resultados: se evidenció una disminución del dolor en dos unidades luego de las cuatro semanas de intervención ( $p<0.05)$. Asi mismo, la incapacidad disminuyó tres unidades ( $p<0.05)$. Dichos valores se mantuvieron al cabo de seis meses después de la intervención. Conclusiones: un programa de ejercicio basado en el fortalecimiento de los músculos abdominales, profundos del cuello y el estiramiento de la cadena recta posterior puede disminuir el dolor y la incapacidad laboral en trabajadores de oficina, este efecto se puede mantener hasta seis meses posterior a la intervención. MÉD.UIS. 2017;30(3):83-8.

Palabras Clave: Dolor de la Región Lumbar. Servicios de Salud del Trabajador. Modalidades de Fisioterapia.

\section{Effects of an exercise plan on low back pain in office workers}

\section{ABSTRACT}

Introduction: lumbar pain is a condition catalogued as one with the higher rate of medical services consultation. In office workers there is also a high prevalence, thus is necessary to generate intervention plans since primary intervention encourage improvement in health status of this population. Objective: to determine the effects of an exercise plan on low back pain in office workers. Material and method: it was conducted an exercise plan in the office based in the strengthening of the abdominal girdle, the neck's deep muscles and the stretching of back straight chain in 17 workers of a company located in Bogota, for four weeks, and evolution respect to low back pain was evaluated through the Roland Morris questionnaire. Tools were applied at the end of the four weeks intervention and follow-up at six months was performed. Results: a decrease in pain by two units is evident after four weeks of intervention $(p<0.05)$, this decrease in pain was maintained after six weeks; likewise, the inability decreases in three units $(p<0.05)$ and results are also maintained to track. Conclusion: An exercise plan based in the strengthening of the abdominal girdle, the neck's deep muscles and the stretching of back straight chain reduces pain and disability in office workers, this effect was maintained at 6 months.MÉD.UIS. 2017;30(3):83-8.

Keywords: Low Back Pain. Occupational Health Services. Physical Therapy Modalities.

Artículo recibido el 10 de Octubre de 2016 y aceptado para publicación el 17 de septiembre de 2017 
¿Cómo citar este artículo?: Alfonso-Mora ML, Romero-Ardila YP, Montaño-Gil EM. Efectos de un programa de ejercicios sobre el dolor lumbar en trabajadores de oficina. MÉD.UIS. 2017;30(3):83-8.

\section{INTRODUCCIÓN}

El dolor de espalda en la población mundial es un problema de salud, ubicado como la segunda causa de consulta en los centros asistenciales, se estima que en la población occidental la incidencia de lumbalgia varía entre el $60 \%$ y $90 \%$, siendo afectadas aproximadamente entre $55-80 \%$ de las personas al menos una vez en la vida como consecuencia de esto'. Lo anterior ha generado aumento en la discapacidad crónica y certificados de baja e invalidez derivados del lumbago inespecífico².

El dolor lumbar es causado hasta en $97 \%$ de los casos por alteraciones mecánicas de la columna; en su mayoría se trata de trastornos inespecíficos de origen músculo-ligamentoso y otros menos frecuentes de origen degenerativo o relacionado con la edad, como las hernias de disco en un $4 \%$ o la estenosis del canal vertebral en un 3\%. Tan sólo el $1 \%$ de los casos son atribuibles a causas no mecánicas, como neoplasias o infección, y el $2 \%$ restante se origina en alteraciones viscerales (enfermedades renales, órganos pélvicos, gastrointestinales o aneurismas aórticos, entre otras) que producen dolor referido 3 .

Otras causas desencadenantes del dolor lumbar incluyen factores físicos como el levantamiento de peso, las cargas físicas elevadas, la vibración, el estrés postural, factores psicosociales y ambientales. En cuanto a los factores de riesgo relacionados con el trabajo, se ha indicado que se relaciona con el uso excesivo de la computadora, estar sentado durante más de la mitad de un día de trabajo combinado con posturas forzadas o una posición en flexión anterior de tronco frecuente, además de una pobre ergonomía ${ }^{4}$.

El personal de oficina utiliza frecuentemente el computador, los cuales tienen naturaleza sedentaria ${ }^{5}$, situación que para términos de la columna lumbar implica sobrecarga para el pilar posterior de los segmentos vertebrales, además de la disminución de las cualidades físicas, que también se constituyen como un factor de riesgo, pues la columna y las estructuras adyacentes reducen la capacidad para resistir la fuerza, lo que la predispone a lesiones durante la carga ${ }^{6}$.
Con base a lo anterior, se ha evidenciado que creando programas de intervención que mejoren la biomecánica de la columna lumbar en los que se combinen componentes físicos y educativos relacionados con el trabajo se producen mejoras en la salud de las personas y se relaciona con una mayor productividad y menores reincidencias de incapacidad por lumbalgia”.

El objetivo de este estudio es identificar el efecto en el dolor e incapcidad causada por lumbago en trabajadores de oficina a partir de un plan de ejercicios basado en el fortalecimiento de la musculatura profunda del cuello y de la faja abdominal, más el estiramiento de la cadena recta posterior.

\section{Materiales y métodos}

Estudio prospectivo observacional de enfoque cuantitativo realizado en el año 2015 en un grupo de trabajadores de oficina con dolor lumbar subagudo o crónico, la muestra estuvo constituida por 20 trabajadores de una empresa ubicada en Bogotá. Se incluyeron a personas de ambos géneros con dolor lumbar actual o intermitente durante los últimos seis meses; se excluyeron a las personas con alteraciones estructurales en la columna vertebral como discopatías, hernia discales, espondilolisis, espondilolistesis, escoliosis, al igual que las personas con diagnósticos de cardiopatías, afecciones neuromusculares, síndromes tumorales o cuadros infecciosos agudos 8 , teniendo un total al final de 17 participantes.

Se tomaron datos demográficos y de la historia de salud de cada uno de los participantes. Luego se evaluó la incapacidad funcional relacionada con el dolor lumbar por medio del cuestionario de Ronald Morris ${ }^{9}$, el cual consta de 24 preguntas que indagan sobre las actividades de la vida diaria de la persona, las respuestas son positivas o negativas, dándole al sí un valor de 1. La sumatoria de todos los puntos oscila entre 0 a 24, presentando mayor incapacidad funcional la persona en cuanto mayor sea la valor total obtenido ${ }^{10}$. También se midió el dolor lumbar con la escala análoga verbal del dolor calificándolo de 0 a 10. 
La intervención fue individual aplicando el plan de ejercicios descrito posteriormente con una duración de 15 minutos con cada trabajador en el puesto de trabajo, durante la pausa activa en sus jornadas laborales, la frecuencia fue de cinco veces por semana, en el transcurso de 4 semanas.

Los ejercicios realizados ${ }^{11,12,13}$ se enfocaron en el fortalecimiento de los músculos abdominales descritos por Francoise Mezìerés ${ }^{13}$ mientras se estira la cadena muscular posterior. La postura de la plancha se realizó manteniendo la postura a tolerancia de la persona, cuidando la alineación cervical, la contracción isométrica abdominal mientras los talones se mantenían fijos al suelo para estirar la musculatura posterior (Ver figura 1). Se realizó con una duración de 20 segundos, aumentando progresivamente 15 segundos por semana, al finalizar los 20 días se completaron 180 segundos del mismo durante cada sesión.

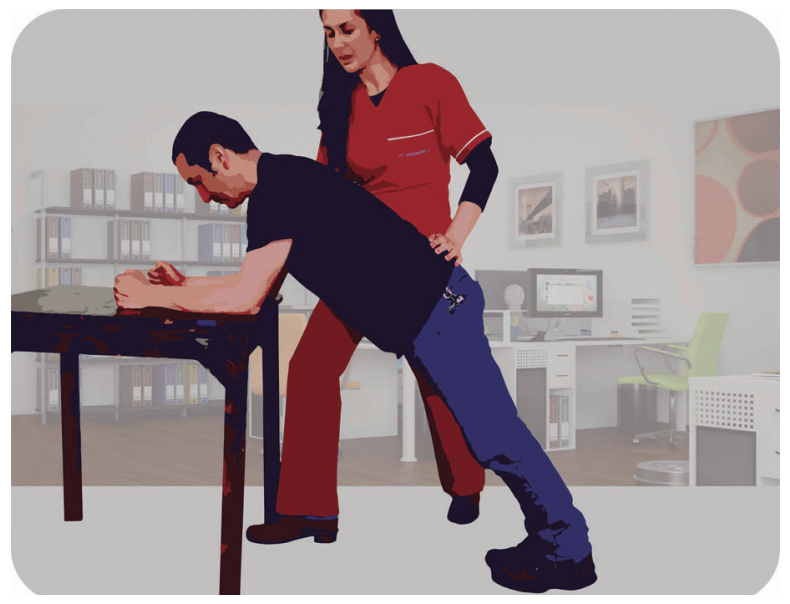

Figura 1. Postura de plancha.

Fuente: Autores.

La postura sedente largo (Ver Figura 2), también pretende estirar la cadena recta posterior, mientras se hace la contracción de los músculos recto abdominal, oblicuos y transverso abdominal. Además, con rotación externa de los hombros y extensión de los codos para facilitar la alineación cervico-dorsal. El primer día se inició con 15 segundos, cada día se aumentó nueve segundos del ejercicio finalizando a las cuatro semanas con 180 segundos de intervención.

Para el fortalecimiento de los músculos profundos del cuello se realizó la contracción isométrica mantenida durante 20 segundos con tres repeticiones con una frecuencia semanal de cinco veces durante las cuatro semanas. Para el ejercicio tres el terapeuta estabiliza el tronco de la persona con una mano y proporciona resistencia con la otra, para la extensión en el occipucio buscando la activación de los músculos recto mayor y menor de la cabeza mas el esplenio del cuello (Ver Figura 3). Se realizó el mismo procedimiento para activación de la porción anterior de los músculos profundos del cuello (largo del cuello largo de la cabeza) haciendo el contacto en el frontal. Estos ejercicios se realizaron durante 10 sesiones de intervención manteniendo una contracción isométrica de 20 segundos con tres repeticiones ${ }^{12}$.

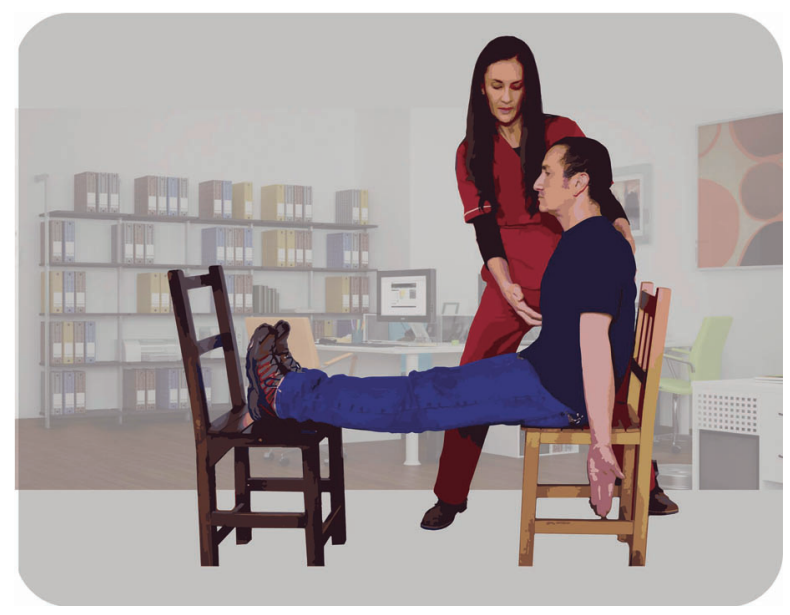

Figura 2. Ejercicio sedente largo.

Fuente: Autores.

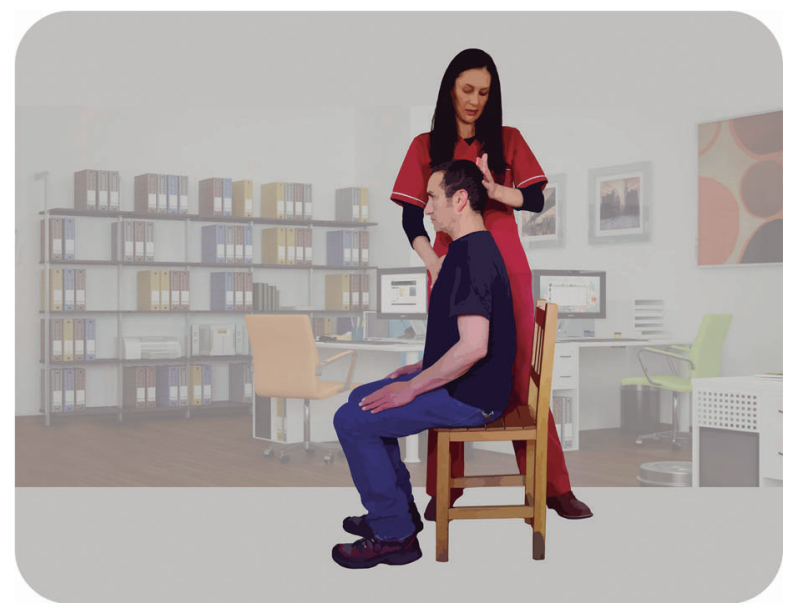

Figura 3. Fortalecimiento de musculatura profunda de la cabeza y el cuello.

Fuente: Autores.

Se finalizó con ejercicios de compresión y tracción de la columna cervical pidiendo al sujeto generar resistencia ante la tracción ejercida pasivamente por parte del fisioterapéuta o la compresión tal como se observa en la figura 4. Este ejercicio se realizó 10 días intercalado con los ejercicios de la figura 3, con tres repeticiones de 20 segundos cada una ${ }^{12}$. 


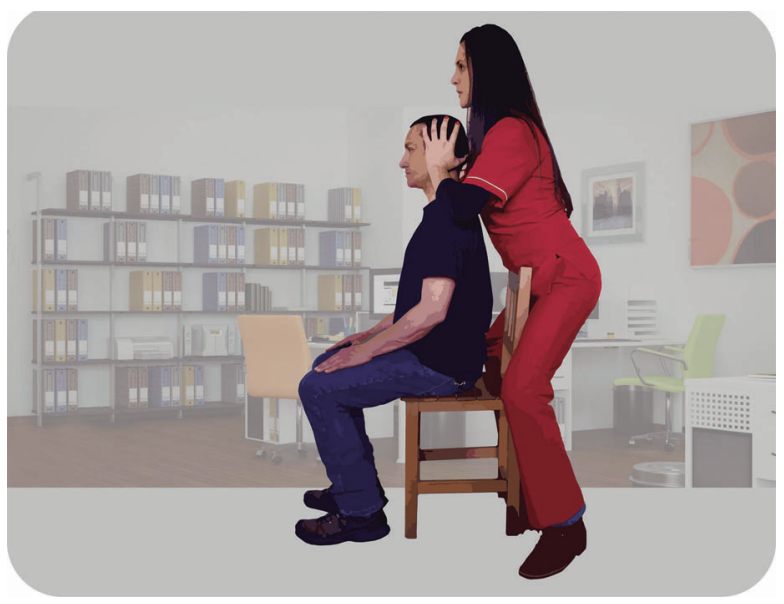

Figura 4. Ejercicios de tracción-compresión Fuente: Autores.

Posterior a las 20 sesiones de intervención se realizó la revaloración a todos los participantes respecto al dolor e incapacidad funcional, $y$ al cabo de los seis meses se realizó el seguimiento. Los datos se registraron en el procesador de Excel y posterior a la depuración de los mismos se aplicaron medidas estadísticas en el programa estadístico SPSS versión 21 de la Universidad de La Sabana. Para la descripción de las variables categóricas se usaron frecuencias relativas y para las númericas promedio con desviación estandar, para la comprobación del cambio en el dolor y la incapacidad se usó la comparación de medianas por medio de la prueba no paramétrica para muestras relacionadas (test de Wilcoxon) y el valor de probabilidad fue menor de 0,05.

\section{ResultadOS}

De los 17 participantes el $76 \%$ eran hombres y el $24 \%$ eran mujeres, quienes se encontraban en un rango de edad de 30 a 40 años. Respecto al cargo que desempeñaban en la empresa, se obtuvo un mayor porcentaje en el área tecnica (Ver Tabla 1).

Por otro lado, la evaluación del dolor de los trabajadores medido con la escala análoga visual demostró un cambio positivo, disminuyendo dos unidades, siendo esto estadísticamente significativo $(p<0,001)$. Dicho cambio se mantuvo seis meses después de realizada la intervención. La comparación de los valores evaluados por el cuestionario de Ronald Morris en el grupo, al inicio y al final de la intervención, disminuyó $(p<0,005)$. Es decir, se evidencia que al disminuir la sintomatología dolorosa en la zona lumbar, se mejora la capacidad de realizar las actividades de la vida diaria (Ver Tabla 2).
Tabla 1. Descriptores demográficos

\begin{tabular}{|l|c|}
\hline \multicolumn{2}{|c|}{ Datos demograficos } \\
\hline Hombres & $24 \%$ \\
\hline Mujeres Cargo en la empresa \\
\hline Promedio de edad & $76 \%$ \\
\hline \multicolumn{2}{|c|}{39 años } \\
\hline Jefe & $6 \%$ \\
\hline Supervisor & $6 \%$ \\
\hline Técnico & $41 \%$ \\
\hline Operario & $17 \%$ \\
\hline Organizador Auxiliar & $18 \%$ \\
\hline Auxiliar & $12 \%$ \\
\hline
\end{tabular}

Fuente: Autores.

\section{Discusión}

De acuerdo con el objetivo del estudio, para determinar los efectos de un protocolo de ejercicio terapéutico sobre la incapacidad y el dolor lumbar se encontró disminución del dolor estadísticamente significativo, lo cual coincide con Houshang A y colaboradores ${ }^{14}$, quienes demostraron que la implementación de un programa de ejercicio, lleva a la disminución de los síntomas relacionados con afecciones del sistema musculoesquelético y en cierta medida genera control sobre los factores psicosociales en el trabajo.

Por otra parte, Lawand P y colaboradores evaluaron el efecto de un programa de estiramiento muscular, utilizando el método de reeducación postural global para las personas con dolor lumbar crónico; donde evidenciaron que el grupo con este tipo de intervención tuvo mejoras estadísticamente significativas $(p<0.05)$ en la escala análoga visual para el dolor y en la incapacidad también medida con el cuestionario Ronald Morris. En mencionado estudio también se reportan cambios en aspectos emocionales, limitación en el funcionamiento físico, la vitalidad y en la calidad de vida relacionada con la salud. Los cambios se mantuvieron durante los siguientes seis meses después de finalizada la intervención ${ }^{15}$; lo que demuestra que los ejercicios globales enfocados, a la corrección postural, activación y estiramiento muscular disminuyen la sintomatología dolorosa en personas con presencia de dolor lumbar.

Merepeza $\mathrm{A}^{16}$ en una revisión sistemática reportó que algunos estudios han encontrado que el tratamiento 
de la lumbalgia crónica con la manipulación espinal, puede disminuir significativamente el dolor y mejorar la función, asímismo el ejercicio terapéutico también ha demostrado que proporciona una mejora en el dolor y funcionalidad, sin embargo, ninguno de estos estudios han reportado intervención en población trabajadora de oficina, por tanto se necesitan más estudios como ensayos clínicos aleatorizados con diseños más rigurosos y poblaciones más amplias, para determinar la efectividad del ejercicio terapéutico sobre el dolor lumbar en personas trabajadoras.

Tabla 2. Cambios en el dolor e incapacidad laboral

\begin{tabular}{|c|c|c|c|c|c|c|c|}
\hline \multirow{2}{*}{ variable } & \multicolumn{2}{|c|}{ Mediana } & \multirow{2}{*}{ Dif } & \multirow{2}{*}{$\mathbf{p}$} & \multirow{2}{*}{$\begin{array}{c}\text { Seguimiento } 6 \\
\text { meses }\end{array}$} & \multirow{2}{*}{ Dif } & \multirow{2}{*}{$p$} \\
\hline & Inicial & Final & & & & & \\
\hline Incapacidad & 5,4 & 3,1 & 2,3 & $0.005^{*}$ & 2 & 3.4 & $0.004^{*}$ \\
\hline dolor & 4,1 & 1,5 & 2,6 & $0.001^{*}$ & 0 & 4.1 & $0.001 *$ \\
\hline
\end{tabular}

${ }^{*} p<0,05$ : diferencia estadísticamente significativa. Dif: unidades de cambio del dolor e incapacidad respecto al inicio y final de la intervención. Fuente: Autores.

La incapacidad en el presente estudio mostró un cambio significativo, lo que se relaciona con una mejora en la realización de las actividades laborales y cotidianas de cada uno de los trabajadores; este hallazgo también fue reportado por Renovato y colaboradores $^{17}$, quienes determinaron los efectos positivos del estiramiento y la estabilización segmental en la discapacidad funcional en pacientes con dolor lumbar crónico. Los efectos de los ejercicios de estabilización segmentaria fueron superiores a los efectos del estiramiento muscular para las variables medidas asociadas con el dolor crónico de espalda baja en comparación con el valor inicial, respecto a la discapacidad $(p<0,01)$.

Por otro lado, Falla ${ }^{18}$ manifiesta que tras la intervención con un programa de ejercicio dirigido al entrenamiento de los músculos flexores craneocervicales, en personas con dolor de cuello crónico, se encuentra mayor capacidad para mantener una postura neutral de cuello prolongando más allá de la sesión, lo que también genera una contribución en la disminución de cargas sobre la zona lumbar y adicionalmente menor dolor en ambas regiones de la columna vertebral.

Finalmente, se evidencia una limitación del estudio realizado, dado a que la muestra no es significativa; por tanto, es importante establecer nuevos estudios para documentar plenamente la influencia del ejercicio correctivo a largo y corto plazo sobre la mejora de la alineación postural y movimiento funcional, evitando lesiones musculoesqueléticas para minimizar el dolor en trabajadores ${ }^{19}$.

\section{CONCLUSIONES}

Los resultados evidencian que los ejercicios aplicados en un grupo de trabajadores con lumbalgia mecánica pueden generar cambios en el dolor y la incapacidad funcional. Por tanto, incluir esta propuesta de fortalecimiento muscular basado en cadenas musculares puede ser efectivo para los planes de atención de salud de los trabajadores desde prevención primaria y secundaria, lo que determinaría un impacto en la disminución de la incidencia de lumbago e incapacidades por esta causa.

\section{FINANCIACIÓN}

Trabajo de investigación financiado con recursos propios.

\section{CONFLICTO DE INTERESES}

Ninguno de los autores declara conflicto de intereses para la publicación de este artículo.

\section{CONSIDERACIONES Éticas}

En todo el proceso de investigación se respetó la dignidad de las personas y se protegió su privacidad. A los participantes se les informó el propósito y beneficios del estudio mediante el consentimiento informado, en coherencia con la Resolución 8430 de 1993 del Ministerio de Salud de Colombia y con 
el aval del grupo de investigación en movimiento corporal humano del programa de fisioterapia de la Universidad de La Sabana.

\section{Agradecimientos}

Las autoras agradecen a la Universidad de La Sabana y a la Empresa Angelcom por su apoyo en el proceso.

\section{REFERENCIAS BIBLIOGRAFICAS}

1. Duque I, Zuluaga D, Pinilla Ana. Prevalencia de lumbalgia y factores de riesgo en enfermeros y auxiliares de la ciudad de Manizales. Promoción Salud. 2011;6(1):27-38.

2. Palomino B, Jiménez L, Ferrero A. El dolor lumbar en el año 2009. Rehabilitación. 2010;44(1):69-81 .

3. Cardenas R. Dolor lumbar: una aproximación general basada en la evidencia. Univ. Méd. Bogotá. 2008;49(4):509-20.

4. Janwantanakul P, Pensri P, Moolkay P, Jiamjarasrangsi W. Development of a risk score for low back pain in office workers - a cross-sectional study. BMC Musculoskeletal Disorders. 2011;12(23):2-8

5. Vidarte J, Vélez C, Sandoval C, Alfonso M. Actividad física: estrategia de promoción de la salud. Promoción Salud 2011;16(1):202-18.

6. Prawit J, Sitthipornvorakul E, Paksaichol A. Risk factors for the onset of nonspecific low back pain in office workers: a systematic review of prospective cohort studies. J Manipulative Physiol Ther. 2012;35(7):568-77.

7. Del Pozo B, Gusi N, Adsuar JC, del Pozo J, Parraca JA, HernandezMocholí M. Musculoskeletal fitness and health-related quality of life characteristics among sedentary office workers affected by sub-acute, non-specific low back pain: a cross-sectional study. Physiotherapy. 2013:99(3):194-200.
8. Hernández R, Fernández C, Baptista MP. Metodología de la investigación. 5th ed. Mexico:Mc Graw Hill. 2010.

9. Roland M, Morris RW. A study of the natural history of back pain. Part I: Development of a reliable and sensitive measure of disability in low back pain. Spine. 1983;8(2):141-4.

10. Guc E, Galdames S, Rebolledo P. Adaptación cultural y validación de la versión chilena del Cuestionario de Discapacidad RolandMorris. Rev méd Chile. 2014;142(6):716- 22.

11. Falla D, Jull G, Russell T, Vicenzino B, Hodges P. Effect of neck exercise on sitting posture in patients with chronic neck pain. Phys Ther. 2007;87(4):408-17.

12. Schomacher J, Erlenwein J, Dieterich A, Petzke F, Falla D. Can neck exercises enhance the activation of the semispinalis cervicis relative to the splenius capitis at specific spinal levels?. Man Ther. 2015;20(5):694-702.

13. Cittone JM. Méthode Mézières. Encycl Méd Chir. 1999;8:1-7.

14. Houshang A, Heydari M, Jalil S, Mostaghaci M, Hossein M, Taheri M. Ergonomic intervention, workplace exercises and musculoskeletal complaints: a comparative study. Med J Islam Repub Iran. 2014;16(1):28-69.

15. Lawand P, Lombardi I, Jones A, Sardim C, Ribeiro LH, Natour J. Effect of a muscle stretching program using the global postural reeducation method for patients with chronic low back pain: A randomized controlled trial. Joint Bone Spine. 2015;82(4):272-7.

16. Merepeza A. Effects of spinal manipulation versus therapeutic exercise on adults with chronic low back pain: a literature review. J Can Chiropr Assoc. 2014;58:456-66.

17. França FR, Burke TN, Caffaro RR, Ramos LA, Marques AP. Effects of muscular stretching and segmental stabilization on functional disability and pain in patients with chronic low back pain: a randomized, controlled trial. J Manipulative Physiol Ther. 2012;35(4):279-85.

18. Falla D, Jull G, Russell T, Vicenzino B, Hodges P. Effect of neck exercise on sitting posture in patients with chronic neck pain. Phys Ther. 2007;87(4):408-17.

19. Rencher N, George J, Vehrs P, Ridge S, Fellingham G. The Acute Effects of Whole-Body Corrective Exercise on Postural Alignment. Int J Exerc Sci. 2015;8(3):213-23. 